\title{
ConsensusPrime - a bioinformatic pipeline for alignment filtering and ideal consensus primer design
}

Maximilian Collatz ( $\square$ maximilian.collatz@leibniz-ipht.de)

Leibniz Institute of Photonic Technology (IPHT), 07743 Jena, Germany

Sascha D. Braun

Leibniz Institute of Photonic Technology (IPHT), 07743 Jena, Germany

Stefan Monecke

Leibniz Institute of Photonic Technology (IPHT), 07743 Jena, Germany

Ralf Ehricht

Leibniz Institute of Photonic Technology (IPHT), 07743 Jena, Germany

\section{Research Article}

Keywords: ConsensusPrime, molecular, sequence, homogeneous

Posted Date: August 25th, 2022

DOI: https://doi.org/10.21203/rs.3.rs-1030641/v2

License: (c) (i) This work is licensed under a Creative Commons Attribution 4.0 International License. Read Full License 


\section{Abstract}

High quality oligonucleotides for molecular amplification and detection procedures of diverse target sequences depend on sequence homology. The processing of input sequences and the identification of homogeneous regions in alignments can be done manually only if alignments are small and if they contain sequences of high similarity. For large and inhomogeneous alignments, the process of finding the best regions needs to be automated.

The ConsensusPrime pipeline was developed sort out redundant and technical interfering data in multiple sequence alignments and detect the most homolog regions from multiple sequences. It automates the prediction of optimal consensus primers for molecular analytical and sequence-based procedures/assays.

ConsensusPrime is a fast and easy to use pipeline for predicting optimal consensus primers that is executable on local systems without depending on external resources and webservices. An implementation in a Docker image ensures platform-independent executability and installability despite the combination of multiple programs. The source code and installation instructions are publicly available on GitHub (https://github.com/mcollatz/ConsensusPrime).

\section{Introduction}

The basic unit of organization of all living organisms is the cell, most cells carry a genome which encodes all of its properties in their DNA. In the recent 20 years, it has become feasible to read these genomes as a whole. Comparison and analysis of complete genomes is now becoming the gold standard for genotyping, and knowledge of full-length genome sequences is becoming the basis for developing various molecular assays for detection and typing. DNA is, compared to other biologically relevant macromolecules, a long-term stable molecule and can be amplified exponentially (PCR, isothermal amplifications) enabling highly sensitive assay formats. The specific Watson-Crick base pair binding in DNA - DNA and DNA - RNA hybridizations allows a highly specific detection of nucleic acid sequences even in very low concentration ranges and under stringent conditions. Therefore, molecular assays can easily be developed, manufactured, stored, delivered, multiplexed and compared between different labs with a reasonable and constantly dropping price per test. But there are also challenges in the assay development. The design of oligonucleotide primers and/or probes is a crucial task for many of these above-mentioned biomedical studies and molecular applications aiming, e.g., on the identification of pathogenic organisms and their resistance and virulence genes. Other applications benefitting from highquality consensus oligonucleotides include FISH analysis, Northern blots, microarray based analyses, isothermal amplification procedures, and all types of molecular assays utilizing linear or exponential amplifications (Mullis 1990). To ensure the widespread usability of oligonucleotide sequences, the detection of high-quality consensus regions among relevant target sequences is a necessary prerequisite. Given the inherent variability of, e.g., bacterial or viral target genes and given the massive 
increase of the number of published target sequences, it becomes necessary to automatize the process of aligning these sequences and detecting suitable consensus regions.

For the performance and applicability of predicted primers, the selection of the target sequences that serve as the basis for the alignment plays a decisive role. This is because functional oligonucleotide sequences can only be predicted if the selected sequences are also representative for the sequences to be examined in a given sample type. The basis for a representative consensus sequence is a high-quality multiple sequence alignment. The alignment quality depends on several factors such as type and length of the input sequences, parameters like gap opening/extension costs and on the algorithm itself. Therefore, the parameters for calculating the alignments vary based on the particular requirements of the alignment.

\section{Design And Implementation}

As the entire pipeline is based on multiple sequence alignments. Their quality is of great importance for functional assays. Therefore, the parameters of MAFFT (Nakamura, Yamada et al. 2018) were adjusted to precisely fulfill the alignment requirements in every alignment step. This is of particular importance when aligning the short primer sequences for visualization. In this case the - - adda gments parameter of MAFFT is used to properly align the short primers to their origin. MAFFT also allows the automated adjustment of the strand direction of a sequence. Another important parameter is - - adjustdirectio $\in$ that allows the automated detection and adjustment of the strand direction in which sequences are provided, as well as the mapping of the reverse primers.

To avoid unwanted distortions of the consensus score(s) due to overrepresented sequences, identical sequences are removed from the alignment, unless specified differently using the - - keepduplicates parameter. The - - consensussimilarity parameter defines the similarity cutoff for each sequence in comparison to the consensus sequence of the alignment. The default value of, e.g., 0.8 means that a sequence has to have at least $80 \%$ of its aligned nucleotides in common with the consensus sequence. Otherwise the sequence is removed from the alignment for primer prediction. To identify ideal consensus oligos, the consensus sequence is re-calculated on the filtered alignment. Therefore, the pipeline uses MAFFT to align all input sequences together in a global multiple sequence alignment and it identifies for every position in the alignment the most common nucleotide. In addition, a consensus score is calculated for every alignment position which is the ratio of the respective count/number of most common nucleotide or gap symbol (-) at that position to the total number of sequences. All letters that are not ATGC are treated as gap. A perfectly conserved region in which all sequences at a given position are identical is thus assigned a consensus score of 1 . The pipeline allows the user to control the quality values of the consensus sequence used for primer prediction via the - parameter. The default value of 0.95 ensures that the most abundant nucleotide occurs in at least $95 \%$ of the sequences at the given position. In addition, the regions above the threshold must have a contiguous minimum length of at least 20 nucleotides. All regions that fall below these values are excluded from the subsequent primer prediction. 
Before the consensus regions are identified for primer design, any gaps are removed from the consensus sequence as well as the corresponding value from the consensus scores. This is necessary because gaps are not encoded by nucleotides and are therefore not relevant for primer design. Gaps in the consensus sequence are caused by insertions in one or more related sequences of the alignment for better visualization.

From this "gapless consensus sequence" the regions relevant for primer design are identified.

As Primer3 (Untergasser, Cutcutache et al. 2012) searches for the primer pair in a contiguous sequence section, instead of using the area in which primers are to be searched (SEQUENCE_INCLUDED_REGION and SEQUENCE_INTERNAL_INCLUDED_REGION), all areas in which primers are not to be searched are excluded by the pipeline (SEQUENCE_EXCLUDED_REGION and SEQUENCE_INTERNAL_EXCLUDED_REGION). This allows the prediction of primers in non-consecutive sequence segments. Furthermore, the gapless consensus sequence is automatically written into the primer3 parameter file (SEQUENCE_TEMPLATE). All other parameters such as melting temperatures or primer lengths are taken from the user-defined Primer3 parameter file. (For a detailed parameter description check out the primer3 manual (https://primer3.org/manual.html). From this, Primer3 predicts the optimal consensus primers and displays the results in a plain text file. The ConsensusPrime pipeline reads the Primer3 output and creates a comprehensive output including all details of previous filter steps in .html format. The predicted primers are added to a final alignment to be visualized using ClustalX (Larkin, Blackshields et al. 2007) and can be manually inspected for logical errors. Note that the reverse primer is added to the final alignment as the reverse complementary sequence.

\section{Results And Discussion}

Unfortunately, automatically assembled sequence alignments often contain a large number of identical sequences as well as partial/fragmented sequences. To limit their influence on optimized primer prediction, the ConsensusPrime pipeline includes several automatic filtering and correction options. First, identical sequences are removed and only a single copy is retained. Another important filtering option is to filter sequences based on their similarity to the consensus sequence. In other words, sequences that are very different from the majority of aligned sequences are removed. This includes not only sequences with a deviating nucleotide composition, but also partial sequences or sequences that contain large insertions compared to the consensus sequence of the input alignment. Short subsequences and also very different sequences are very often found in automatically generated alignments in frequently used databases, which makes extensive filtering methods absolutely essential to ensure a good data basis for the following analyses.

The ConsensusPrime pipeline allows automatic detection of optimal consensus regions from large alignments with many sequences. The necessary alignment filter steps and functions are integral components of the pipeline and ensure an optimal input for the subsequent primer predictions. A summary of the individual filter steps is provided in the created HTML overview. In addition, all individual steps are saved as an alignment to ensure perfect traceability and evaluability. This is of particular 
interest for applications such as the prediction of real-time PCR primer and probe pairs within regions of high homology. Adjustable physiochemical parameters can be set for the design of hundreds of sequences for DNA-based microarray analyses or sequences for the design of other molecular-based analyses (e.g., FISH, molecular beacon technology or isothermal amplifications). All parameters are defined in a simple text file, and therefore, the run parameters can be easily reused or adapted. All adjustments and parameters are summarized in the HTML overview for complete reproducibility. For a clear visualization and inspection of the predicted primers, a final alignment is created. This contains the input sequences used, the consensus sequence, the consensus regions representing the regions used for primer prediction and all predicted primers. For an example, see Fig 1.

The pipeline starts by aligning the input sequences in a multiple sequence alignment and the regions with the best homology are identified. These regions are then used for primer prediction.

Our new pipeline combines custom alignment filters with MAFFT (v7.453) (Nakamura, Yamada et al. 2018) and Primer3 (v2.5.0) (Untergasser, Cutcutache et al. 2012), two common and long-established tools to ensure high-quality alignments and, respectively, primer predictions. An overview of the pipeline is given as a flow diagram in Fig 2.

\section{Availability And Future Directions}

The source code of the pipeline is written in python and easily executable from the Linux command line. The source code and installation instructions are publicly available on GitHub under the MIT license, which allows public and private use as well as modification and distribution of the code (https://github.com/mcollatz/ConsensusPrime). The pipeline is also integrated into a ready-to-use Docker container with all dependencies and necessary programs pre-installed (mcollatz/consensusprime:1.0).

Embedding this pipeline in a Docker container ensures executability on a wide variety of systems. The ConsensusPrime pipeline is not available as an online service to avoid any privacy concerns arising from the transfer of data to third parties and to ensure usability at all times.

\section{Declarations}

Funding

ADA (13GW0456C: BMBF): Adaptable decentralized diagnostics for veterinary and human medicine.

\section{Authors' contributions}


MC implemented the pipeline and wrote the manuscript. SB, SM and RE helped with conceptual development and definition of the requirements

All authors revised and corrected the various drafts and approved the final version of the manuscript.

\section{References}

Larkin, M. A., G. Blackshields, N. P. Brown, R. Chenna, P. A. McGettigan, H. McWilliam, F. Valentin, I. M. Wallace, A. Wilm, R. Lopez, J. D. Thompson, T. J. Gibson and D. G. Higgins (2007). "Clustal W and Clustal X version 2.0." Bioinformatics 23(21): 2947-2948.

Mullis, K. B. (1990). "The unusual origin of the polymerase chain reaction." Sci Am 262(4): 56-61, 64-55.

Nakamura, T., K. D. Yamada, K. Tomii and K. Katoh (2018). "Parallelization of MAFFT for large-scale multiple sequence alignments." Bioinformatics 34(14): 2490-2492.

Untergasser, A., I. Cutcutache, T. Koressaar, J. Ye, B. C. Faircloth, M. Remm and S. G. Rozen (2012). "Primer3--new capabilities and interfaces." Nucleic Acids Res 40(15): e115.

\section{Figures}

\section{Figure 1}

Section of the final alignment visualized with ClustalX (Larkin, Blackshields et al. 2007). The structure of the alignment is always in the order of: filtered sequences, consensus sequence, sequence parts that are used for the primer prediction, predicted primer/probe pairs. This alignment enables the user to check the alignment of the filtered input sequences and the resulting consensus regions that are considered for the actual primer prediction. It also gives a great overview of the predicted Primers to chose the best pair if multiple predictions have been made. The vanA-A sequence of $P$. apiarius contains many nucleotides that deviate from the consensus sequence. In this case, the user must decide whether this sequence should remain in the alignment or whether it should be removed by adjusting the filter parameters.

\section{Figure 2}

ConsensusPrime workflow with input/output files as well as user parameters. The sequences have to be provided in multi-fasta format. The Primer3 parameter file contains the primer/probe parameters for Primer 3 and the ConsensusPrime parameters that influence the pipeline itself are given via the command line. The ConsensusPrime pipeline processes the input data in several successive filter and alignment steps to identify suitable consensus regions. The regions found in this way are automatically written to 
the Primer3 parameter file and the primer prediction is started using Primer3. Afterwards, the output of Primer3 in addition to the details of the individual filter steps are written to a concise .html file. Predicted primers are also added to a final alignment to be displayed with any alignment visualization tool. 